\title{
Route of Hysterectomy for Benign Disease: Abdominal Hysterectomy
}

\author{
Clarissa Polen-De, MD, Jamie Bakkum-Gamez, MD, and Carrie Langstraat, MD
}

\begin{abstract}
The role of abdominal hysterectomy for benign disease has decreased with time, given the broad application of minimally invasive hysterectomy. Abdominal hysterectomy is indicated when minimally invasive surgery is not feasible due to patient-, disease-, or technical-factors, including uterine size, risk of malignancy, or extensive endometriosis and adhesive disease. Conversion from minimally invasive hysterectomy might also be required when unexpected adhesive disease or intraoperative complications are encountered. Attention to preoperative preparation; surgical planning; and intraoperative setup, exposure, and use of available surgical assistance optimize the success of planned or emergent conversion to abdominal hysterectomy. Finally, intraoperative and advanced pelvic surgical techniques, as well as consultation with colleagues across subspecialties, can be utilized to facilitate safe surgery in patients with complex pelvic diseases or inadvertent intraoperative injuries. ( J GYNECOL SURG 37:116)
\end{abstract}

Keywords: gynecology, surgery, hysterectomy, abdominal hysterectomy, gynecologic surgery

\section{Introduction}

A LTHOUGH THE FIRST ABDOMINAL hysterectomy was performed in 1843, it was 10 years later in 1853 that Ellis Burnham ${ }^{1}$ was the first to successfully complete the surgery without a perioperative demise. The first total abdominal hysterectomy was not performed until almost a decade later, when Richardson (cited by Sutton) ${ }^{1}$ introduced the technique to reduce drainage from the cervical stump and cervical carcinoma. Since that time, substantial progress has been made through the developments of anesthesia, antibiotics, and antiseptics, as well as minimally invasive surgery (MIS) techniques-so much so that there are now limited indications for simple total abdominal hysterectomy (TAH).

\section{Indications}

Most hysterectomies for benign disease can be performed in a minimally invasive fashion and, per the American College of Obstetrics and Gynecologists, should be performed whenever feasible due to the well-documented advantages over abdominal hysterectomies. ${ }^{2}$ Abdominal hysterectomy is indicated when MIS is not feasible due to patient-, disease-, or technical-factors. Indications include uterine size, risk of malignancy, or extensive endometriosis and adhesive disease.
Although an upper limit of uterine size precluding minimally invasive hysterectomy has not been established, several studies have shown successful removal up to 17-18 weeks' size. ${ }^{3,4}$ Although technically feasible, larger specimens can be more-difficult to manipulate intraoperatively via MIS and remove via the vagina, adding operative time to the procedure. Large fibroids within the lower uterine segment or cervix can also limit access to the uterine vessels, sometimes barring a safe minimally invasive approach. The size of the pelvis and vaginal access are additional considerations when considering the feasibility of a MIS hysterectomy.

Important considerations when performing a MIS hysterectomy for uterine smooth-muscle tumors include age of the patient, her menopausal status, and rate of uterine growth. Practitioners should have heightened suspicion for sarcomas in postmenopausal women with enlarging uterine masses. Additionally, providers should counsel patients preoperatively on the low, but real, risk of sarcoma and discuss techniques utilized to limit dissemination if cancer is found. Considerations include transvaginal removal using an endoscopic bag or extension of the periumbilical incision with or without morcellation for safe removal.

Studies evaluating algorithms aimed to increase utilization of minimally invasive hysterectomy have shown that

Department of Obstetrics and Gynecology, Division of Gynecologic Surgery, Mayo Clinic, Rochester, Minnesota, USA. 
history of cesarean delivery or prior laparotomy does not necessarily preclude MIS. In patients with endometriosis or surgical histories involving significant adhesive disease, a laparoscopic evaluation can be considered to determine feasibility of a minimally invasive hysterectomy prior to laparotomy. $^{5,6}$

Gynecologic surgeons should be comfortable with abdominal exploration and $\mathrm{TAH}$ in case they encounter significant adhesive disease or need for emergent conversion to laparotomy. Additionally, the skill-set to perform a type 2 radical hysterectomy in which the ureter is isolated and the uterine artery ligated at the level of the ureter is valuable when approaching patients with large cervical or lower uterine segment fibroids, endometriosis, or abnormal placentation or hemorrhage that requires cesarean hysterectomy.

\section{Preoperative Planning: “Over Prepare and You'll Always Be Prepared!"}

Preoperative preparation includes a patient history, examination, imaging, laboratory work, and the consent process. History of disease, medical comorbidities, and surgical history identify risk factors for a suboptimal outcome and indications for further evaluation prior to surgery. A multidisciplinary team of surgical nurses, physician-assistants, anesthesia providers, and surgeons optimizes patient safety and improve patient education. A team-based evaluation and preoperative planning that incorporates the patient, surgeon, and anesthesia providers in a shared decisionmaking approach will best optimize surgical expectations and outcomes.

Transvaginal ultrasound is frequently utilized to assess etiology of abnormal bleeding, to determine the size of the uterus, and to evaluate the adnexa. Computed tomography of the abdomen, pelvis, and possibly chest can be useful for assessing pelvic masses, whether they be uterine or ovarian, and can help identify lymphadenopathy, upper abdominal disease, or lung metastasis if malignancy is in the differential diagnoses. Additional imaging, such as magnetic resonance imaging, might be indicated in the setting of likely endometriosis, evaluation of a uterine mass, or a planned cesarean hysterectomy to address a morbidly adherent placenta.

In addition, discussions about removal of the cervix, fallopian tubes, and ovaries at the time of hysterectomy are relevant. The 2010 Journal of Obstetrics and Gynaecology Canada supracervical hysterectomy guidelines, ${ }^{7}$ which reviewed available evidence from the Cochrane Library, Medline, ${ }^{\circledR}$ and Embase surrounding the potential benefit of supracervical hysterectomy, noted that removal of the cervix was not associated with decreased sexual function, was not superior for prevention of lower urinary-tract symptoms, and was not superior for preventing peri- and postoperative complications. Patients who are considering supracervical hysterectomy should also be counseled that they might have continued vaginal bleeding and will continue to require cervical cancer screening. ${ }^{7-10}$ Although these data are largely based on randomized trials, the researchers concluded that there is "fair" evidence supporting the recommendations that further studies are still warranted. Patients should be counseled on opportunistic salpingectomy at the time of hysterectomy given the fallopian tubes' role in the pathogenesis of ovarian cancer and limited function following removal of the uterus. ${ }^{11}$ Elective oophorectomy in a premenopausal woman warrants a detailed discussion of the increased risk of all-cause mortality, cardiovascular disease, sexual dysfunction, and osteoporosis versus the potential risk reduction of ovarian cancer, breast cancer, and need for future ovarian surgery. ${ }^{12}$

Finally, the informed consent process is not only critical from a shared decision-making perspective, but also provides a framework for discussions about perioperative risks, mitigation of these risks, and how complications will be addressed.

\section{Intraoperative Planning and Anatomical Considerations: The Abdominal Hysterectomy Technique}

Knowledge and understanding of pelvic and abdominal anatomy are of paramount importance to the gynecologic surgeon. Operative techniques used to expose, visualize, and isolate structures during surgery include appropriate use of retractors, packing, and assistants to set the surgeon up for success. Positioning the patient in either a supine or synchronous position with yellow-fin stirrups may be according to provider preference and dependent on surgical need. Supine positioning could decrease the risk of nerve injury, compared to synchronous positioning, but make intraoperative access to the vagina and rectum more difficult should this be required.

Upon entry, the gynecologic surgeon aims to restore normal pelvic anatomy, or as closely as possible, to be able to place an abdominal retractor and pack the bowel away from the surgical field. Various self-retaining abdominalwall retractors are available to improve visualization of the target anatomy and minimize surgical-assistant fatigue, including the Bookwalter, ${ }^{\circledR}$ Balfour, and Omni ${ }^{\circledR}$ retracting systems. A surgeon's preference may be influenced by retractor availability and familiarity, but can also be determined by patient body habitus. Careful placement of retractor blades should be ensured to limit potential injury to the femoral nerve. Additionally, bowel packing generally with laparotomy sponges or a long abdominal pack is an art of its own, with surgeons generally developing techniques that will ensure sustained, adequate exposure of the anatomy while safely protecting the bowel and mesentery. Finally, optimizing utilization of available surgical assistants aids in the ease, efficiency, and success of the procedure significantly.

Although not frequently utilized for benign hysterectomy, the anatomy and technique utilized during a type 2 radical hysterectomy can be useful during a difficult pelvic dissection. ${ }^{13}$ Through a low midline or Pfannenstiel incision, the pelvis is explored, the bowel is packed, a retractor is placed, and the patient is placed in a Trendelenberg position. Traction is placed on the uterus with Kocher clamps on the cornu; the uterus is brought to the patient's left; and the right round ligament is clamped, cut, and suture-ligated. The broad ligament is then incised parallel and lateral to the infundibulopelvic ligament (Fig. 1). With the broad ligament open, the pararectal space is developed with the surgeon's index finger and/or Russian forceps by 


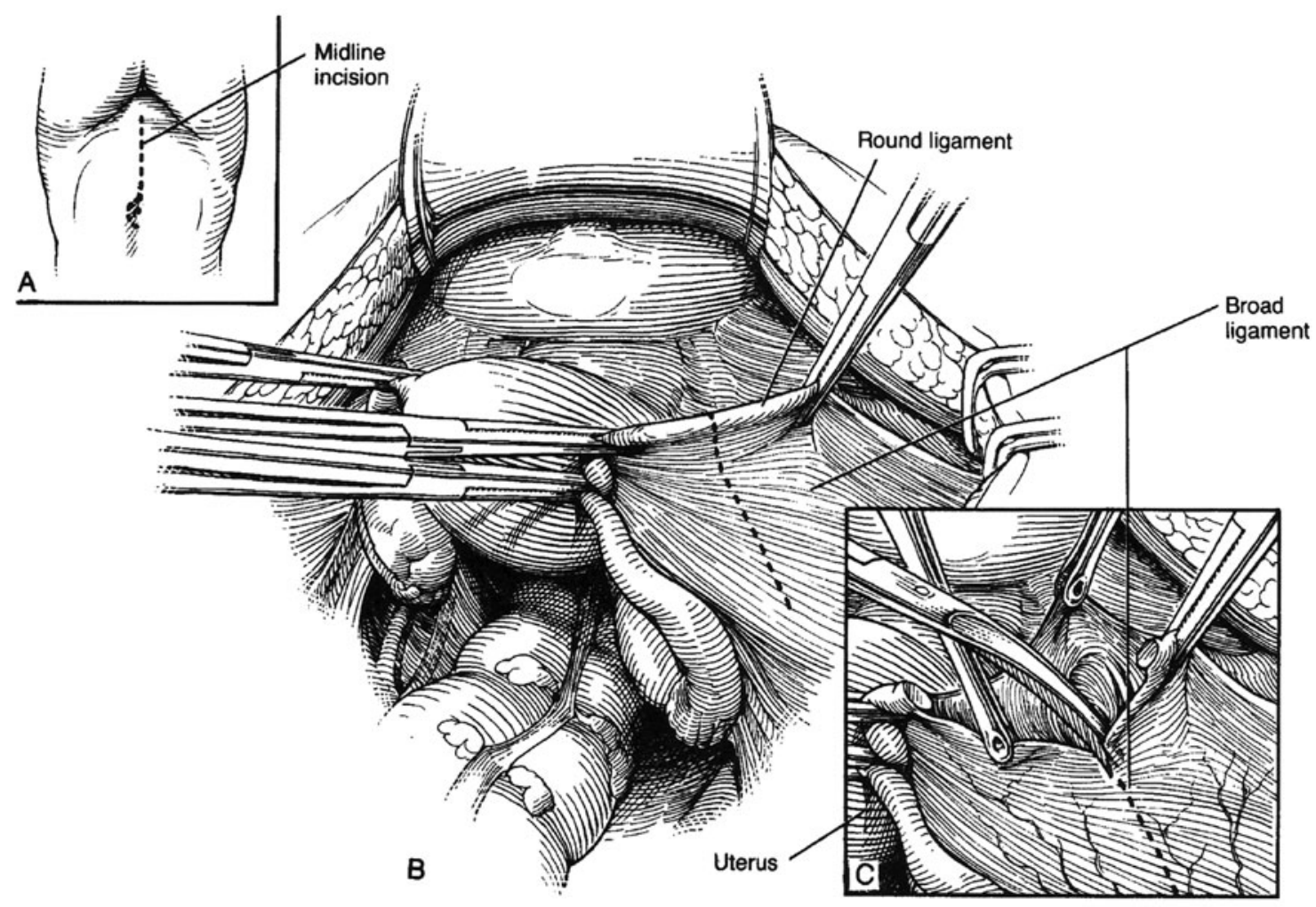

FIG. 1. Entry into the avascular retroperitoneal spaces. Anatomical images obtained from the Atlas of Gynecologic Surgery, by Raymond A. Lee, Philadelphia: W.B. Saunders, 1992. Used with permission of the Mayo Foundation for Medical Education and Research. All rights reserved.
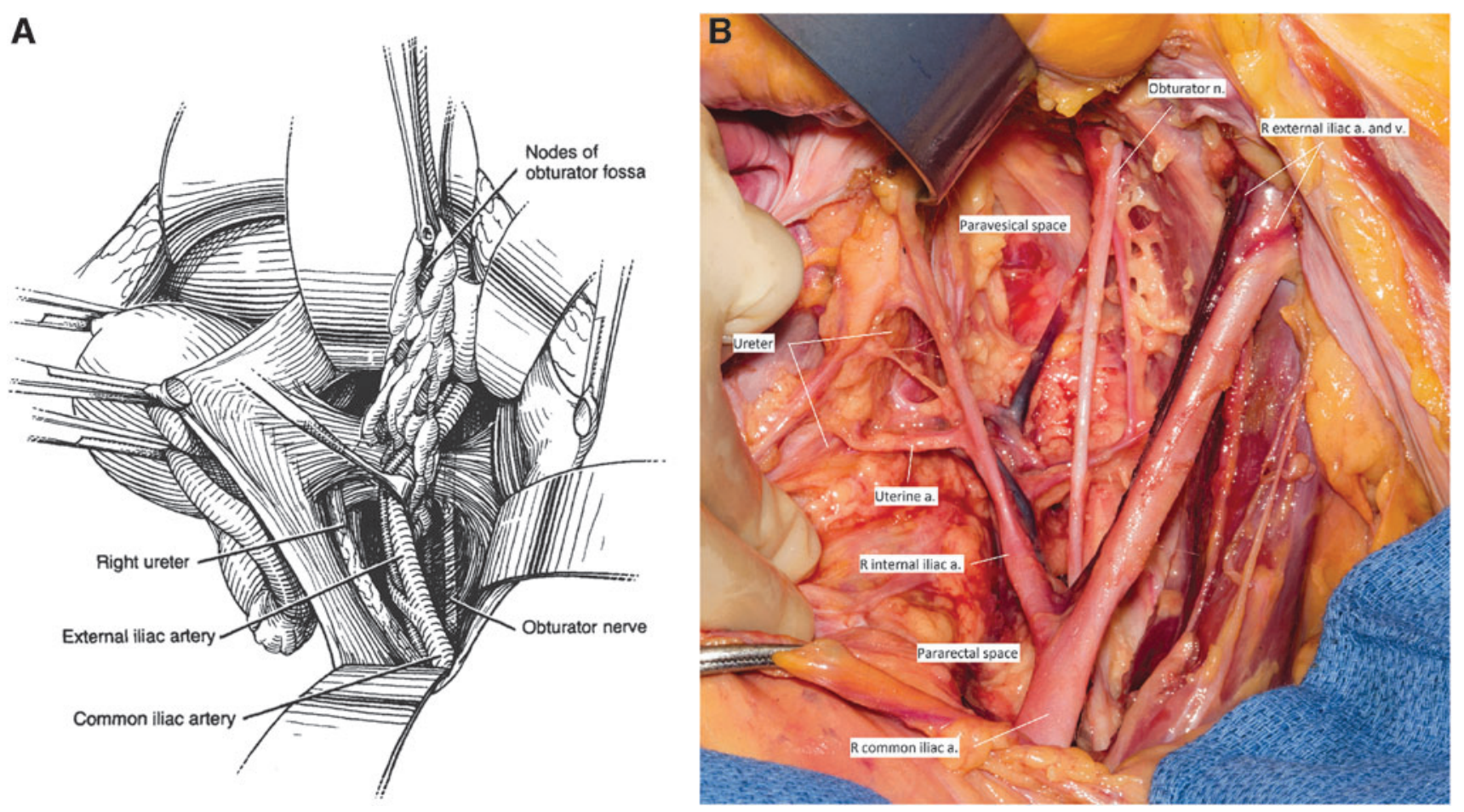

FIG. 2. Right retroperitoneal space depiction (A) and cadaveric dissection (B). Anatomical images obtained from the Atlas of Gynecologic Surgery, by Raymond A. Lee, Philadelphia: W.B. Saunders, 1992. Used with permission of Mayo Foundation for Medical Education and Research. All rights reserved. 
displacement of the pelvic peritoneum and attached ureter medially while keeping the internal iliac artery lateral. The posterior pararectal space is then dissected down to the hollow of the sacrum with care not to disrupt the sacral venous plexus. Moving anteriorly along the internal iliac artery, the obliterated umbilical vessels are identified, displaced medially, and the paravesical space can be de- veloped with an anterior, medial sweeping motion toward the pubic symphysis. Opening pararectal and paravesical spaces enables excellent visualization of the ureter and important vascular structures in the pelvis (Fig. 2).

Next, the ureter should be mobilized from the pelvic peritoneum. The pelvic peritoneum adjacent to the ureter is grasped to provide traction. Using a right angle clamp and
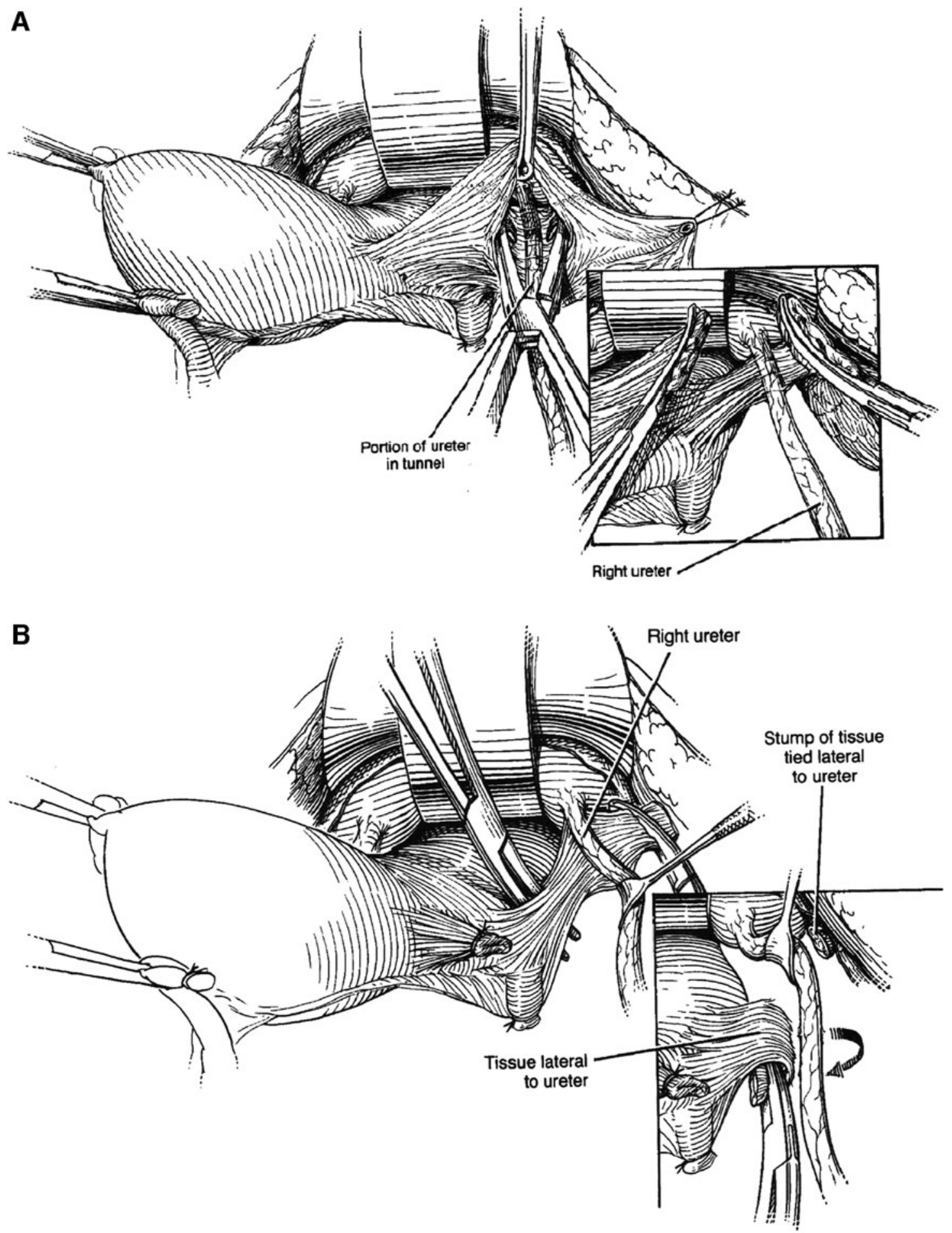

FIG. 3. Ureterolysis (A) and lateralization of the ureter (B). Anatomical images obtained from the Atlas of Gynecologic Surgery, by Raymond A. Lee, Philadelphia: W.B. Saunders, 1992. Used with permission of the Mayo Foundation for Medical Education and Research. All rights reserved. 
fine scissors, the ureter should be freed from the pelvic peritoneum, while being careful not to injure the ureter. The path of the ureter is then followed to its entry into the cardinal ligament complex deep to the uterine artery. A rightangle clamp is used to isolate and clamp the uterine artery (Fig. 3).

The technique utilized in the modified radical hysterectomy enables isolation of critical structures as well as subsequent improved mobility and visualization in difficult pelvic dissections.

\section{If You Get into Trouble: Conversion to TAH}

Despite an optimized surgical plan, intraoperative findings precluding MIS or acute surgical complications can develop necessitating conversion from minimally invasive to open hysterectomy. When planning for intraoperative conversion, the surgical principles above hold true, and conversion to a technique offering improved exposure and visualization should be made. This includes situations requiring conversion of a total vaginal hysterectomy to laparoscopic-assisted or open hysterectomy and conversion of laparoscopic or robotic hysterectomy to open hysterectomy.

The first step for converting to an abdominal hysterectomy is determining the incision type. A vertical midline incision offers superior exposure and is preferable if additional surgical consultation is required. A Pfannenstiel incision offers improved cosmesis with a reduced incidence of a subsequent incisional hernia, but enables only limited extension to improve exposure. A Maylard or Mackenrodt incision is different from a Pfannenstiel incision in that the former is made higher on the abdomen (5-8 $\mathrm{cm}$ above the pubic symphysis) than the latter, but requires that the anterior rectus sheath not be separated from the rectus abdominis muscles, ligation of the inferior epigastric vessels, and, subsequently, transverse transection of the rectus muscles. Although exposure can be improved over that of the Pfannenstiel incision, the Maylard or Mackenrodt incision arguably does not improve cosmesis and essentially eliminates the rectus abdominis muscle as a future flap source for patients with complex surgical needs. A Cherney incision utilizes a low transverse skin incision and transverse incision of the rectus muscles at the tendinous insertion to the pubic symphysis. The Cherney incision results in similar cosmesis to the Pfannenstiel incision and can be considered a as conversion from the Pfannenstiel incision, but might result in retraction of the rectus muscles or pain at the pubic symphysis if repaired. An additional option is a vertical paramedian incision. This incision is thought to result in fewer hernias with similar exposure as the vertical midline incision but could put patients at risk for tissue necrosis if a future midline vertical incision is required.

Intraoperative communication and familiarity with available resources and intraoperative tools are key in cases of emergency conversions to laparotomies. Involving anesthesia colleagues early and requesting assistance from available backups, such as gynecologic oncology, general surgery, urology, or vascular surgery could be required. In the setting of vascular injury, the surgeon can place gentle pressure on the source while calling for help and retaining a calm and controlled operating room. Knowledge of the availability and appropriate use of blood products, hemostatic agents, tranexamic acid, and the possibility for interventional radiology embolization are critical in the setting of hemorrhage. Additionally, surgical control via ligation of the uterine artery, as in the above approach, or ligation of the anterior division of the internal iliac artery can be performed by a surgeon who is comfortable with this technique. In very rare circumstances, pelvic packing with temporary abdominal closure to enable stabilization is an option in the gynecologic surgeon's toolkit. Emergent situations reinforce the need to always be prepared for possible conversion as well as the importance of preoperative preparation, knowledge of available resources, and the ability to utilize these in the setting of rapid intraoperative needs.

\section{Conclusions and Take-Home Points}

Abdominal hysterectomy has decreased given the broad application of minimally invasive hysterectomy for benign disease. Abdominal hysterectomy is indicated when MIS is not feasible due to patient-, disease-, or technicalfactors, including uterine size, risk of malignancy, or extensive endometriosis and adhesive disease. Attention to preoperative preparation, surgical planning, intraoperative assistance, and communication optimize the success of planned or emergent conversion to abdominal hysterectomy and advanced pelvic surgical techniques can be utilized to facilitate safe surgery in patients with complex diseases.

\section{Author Disclosure Statement}

No financial conflicts of interest exist.

\section{Funding Information}

The authors received no funding for this work.

\section{References}

1. Sutton C. Hysterectomy: A historical perspective. Baillieres Clin Obstet Gynaecol 1997;11:1.

2. American College of Obstetricians and Gynecologists (ACOG). ACOG Committee on Gynecologic Practice. Choosing the Route of Hysterectomy for Benign Disease: Committee Opinion 701. Washington, DC: ACOG, 2017.

3. Walters MD, Ridgeway BM. Increasing utilization of minimally invasive hysterectomy. Clin Obstet Gynecol 2017;60:273.

4. Schmitt JJ, Occhino JA, Weaver Al, et al. Vaginal versus robotic hysterectomy for commonly cited relative contraindications to vaginal hysterectomy. J Minim Invasive Gynecol 2017;24:1158.

5. Schmitt JJ, Baker MV, Occhino JA, et al. Prospective implementation and evaluation of a decision-tree algorithm for route of hysterectomy. Obstet Gynecol 2020; 135:761.

6. Schmitt JJ, Carranza Leon DA, Occhino JA, et al. Determining optimal route of hysterectomy for benign indi- 
cations: Clinical decision tree algorithm. Obstet Gynecol 2017;129:130.

7. Kives S, Lefebvre G. Supracervical hysterectomy. J Obstet Gynaecol Can 2010;32:62.

8. Lonnee-Hoffmann R, Pinas I. Effects of hysterectomy on sexual function. Curr Sex Health Rep 2014;6:244.

9. Danesh M, Hamzehgardeshi Z, Moosazadeh M, et al. The effect of hysterectomy on women's sexual function: A narrative review. Med Arch 2015;69:387.

10. Lermann J, Haberle L, Merk S, et al. Comparison of prevalence of hypoactive sexual desire disorder (HSDD) in women after five different hysterectomy procedures. Eur J Obstet Gynecol Reprod Biol 2013;167:210.

11. Semmel DR, Folkins AK, Hirsch MS, et al. Intercepting early pelvic serous carcinoma by routine pathological examination of the fimbria. Mod Pathol 2009;22:985.
12. Erekson EA, Martin DK, Ratner ES. Oophorectomy: The debate between ovarian conservation and elective oophorectomy. Menopause 2013;20:110.

13. Lee RA. Atlas of Gynecologic Surgery. Philadelphia: W.B. Saunders, 1992.

Address correspondence to: Carrie Langstraat, $M D$

Department of Obstetrics and Gynecology Division of Gynecologic Surgery and Medical Oncology

Mayo Clinic

200 First Street Rochester, MN 55905

USA

E-mail: langstraat.carrie@mayo.edi 